
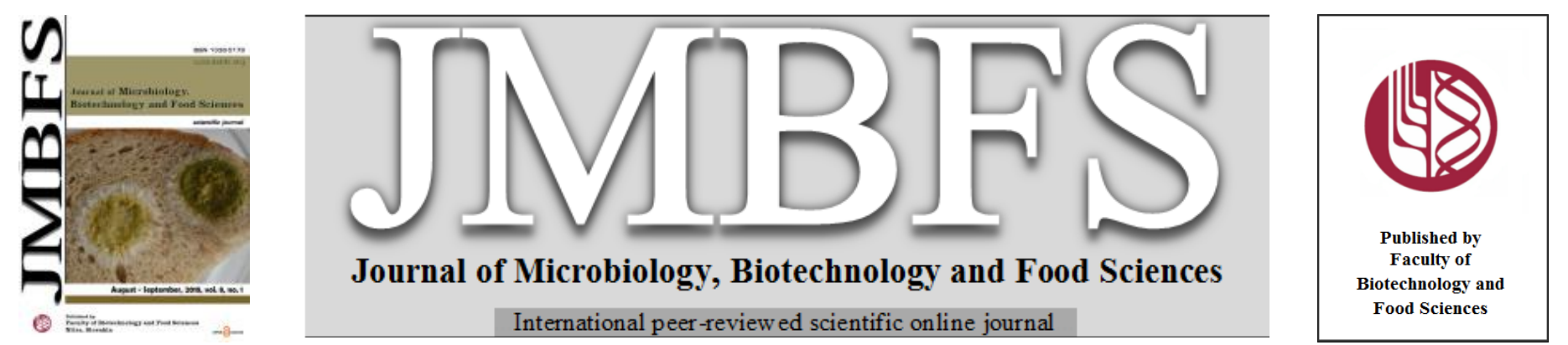

\title{
INACTIVATION OF PISTACHIO CONTAMINANT ASPERGILLUS FLAVUS BY ATMOSPHERIC PRESSURE CAPACITIVE COUPLED PLASMA (AP-CCP)
}

\author{
Mohammad Aminroosta Tasouji ${ }^{1}$, Amir Hossein Ghorashi*2, Mohammad Taghi Hamedmoosavian ${ }^{1}$, Mahdi Bakhshzad Mahmoudi ${ }^{2}$
}

$\operatorname{Address(es):~}$

${ }^{1}$ Chemical Eng. Group, Faculty of Eng., Ferdowsi University, Mashhad, Iran.

${ }^{2}$ Faculty of Plasma \& Nuclear Fusion, Nuclear Science \& Technology Institute, Tehran, Iran.

*Corresponding author: aghorashi@ aeoi.org.ir

doi: $10.15414 / j m b f s .2018 .8 .1 .668-671$

\section{ARTICLE INFO}

Received 15. 11. 2017

Revised 29. 5. 2018

Accepted 13. 6. 2018

Published 1. 8. 2018

Regular article

OPEN $\partial_{\text {ACCESS }}$

\begin{abstract}
Aspergillus flavus toxin contamination of pistachio nuts is a serious problem in terms of health and food security. In this paper, in order to increase the nut shelf life by eliminating or reducing the amount of toxin-producing fungus Aspergillus flavus, pistachios were irradiated with cold plasma, using Atmospheric Pressure Capacitive Coupled Plasma (AP-CCP) generating device using Argon gas. The advantages of this device are both its mobility, adjoining a nuts carrying container for thorough irradiation of the nuts and that the device can be designed in the form of an array of torches for treatment of large quantities of nuts in open air as against in a vaccum chamber. In this way, the sample of nuts are firstly soaked with fungus and then irradiated in different conditions in terms of power, pressure and time. The analysis of experimental results indicates $4 \log (66.6 \%)$ fungus reductions. This pathogen reduction was observed in 100 Watts, at atmospheric pressure and irradiation duration of 10 minutes.
\end{abstract}

Keywords: Pistachio, Aspergillus flavus, Atmospheric pressure plasma, Plasma Treatment

\section{INTRODUCTION}

Mycotoxin is literally formed from two Greek words "Myco" and "toxin". Mycotoxins are a group of toxic compounds naturally produced by several species of fungi, mainly by Aspergillus flavus and Aspergillus parasitiscus. Aflatoxins are the most significant mycotoxins because of their toxic cancer-causing nature, among which, the aflatoxins B1, B2, G1 and G2 are known as the most important types (Arrus et al., 2005); due to their high risks of contaminating agricultural products, they are considered as potential threat to human health and livelihoods. Aspergillus favours pollution and products containing fat and high carbohydrates like oil seeds and nuts (Martins et al., 2008). Therefore, pistachios, peanuts, corn, wheat, rice, almonds and figs are the main hosts of this fungus, where the contamination risks of unroasted nuts are much higher than the roasted. Pistachio is among the most expensive nuts and many people prefer to consume it fresh Moreover, pistachio as an export product is the source of foreign currency income for those countries cultivating it, like Iran, USA, Turkey, Syria, Greece, etc. For instance, Iran has 440 thousand hectares of pistachio orchards acreage and the economic value of its export to 66 countries is annually about one billion US Dollars. Whilst, stringent national and international regulatory for contamination levels are imposed for these toxins. For instance, in United States, the products that contain less than $20 \mathrm{ppb}$ the total aflatoxin and $15 \mathrm{ppb}$ aflatoxins B1 have permissions of purchase, sale, import and export (Gourama et al., 1995).

In order to suppress fungal growth and reduce mycotoxin formation, many remedies have so far been suggested i.e. chemical, physical, farming and biological methodological remedies. Although these methods can reduce surface fungal contaminations, they often require either non-attainable equipment or expensive chemicals and may also cause unacceptable detrimental effect to edible materials. Furthermore, some remedies may even cause excessive health hazards too. As a result, some methods are considered impractical, costly, not completely effective, harmful, and time consuming for treatment of nuts in big scales.

Plasma is partially ionized gases also known as highly energized fourth state of matter that all or a significant parts of its atoms have lost one or more electrons and are changed to positive ions. In other words, plasma is an electrically conductive environment because of large number of positive and negative ions, having high-energy electrons (Petitpas et al., 2007). When the collision is low (cold plasma) the electron temperature is few thousand Kelvin degrees while the temperature of ions and approximate temperature of plasma is only about room temperature (Lieberman et al., 2005). Non-equilibrium atmospheric pressure plasmas have potential for biomedical applications because they are non-thermal and non-toxic. They can provide reasonable reactivity with a low gas temperature at atmospheric pressure. The devices producing plasma are easy to construct because they do not need any vacuum system and can be operated with various feeding gases under a wide range of driving frequencies. Atmospheric pressure plasma jet devices generate plasma in surrounding air, so they are suitable for direct treatment of targets of any size. Factors affecting the formation of plasma are mainly: Plasma forming gas (Argon, Helium, Nitrogen, Methane, Sulfur hexafluoride etc.), gas flow rate, electric and magnetic fields, shape and arrangement of electrodes and finally, the kind of plasma generating device (Roth, 2000). Aspergillus flavus and aflatoxin reduction/elimination methods with cold plasma are earlier reported (Gadri et al., 2000; Ohkawa et al., 2006; Lee et al., 2006; Schneider et al., 2005; Park et al., 2007). The achieved results reported by the referred research works were all achieved either in longer test duration and/or using different methods and environments. It is worth noting that, P. Basaran et al. reported some effective results on treatment of Aspergillus parasiticus from sampled nut surface with low pressure cold plasma using air gases and Sulfur hexafluoride (SF6) (Moisan et al., 2002; Basaran et al., 2008).

The main objective of the present research work was to explore possible applications of cold plasma, using Argon gas to inactivate/reduce Aspergillus flavus on pistachios as a sample representative of varieties of nuts. The present research has achieved good results by changing the plasma forming gas to Argon, using AP-CCP generating device for the first time. Moreover, Argon has its own advantages such as low cost, less power consumption, and high sterilization efficacy (Samarajeewa et al., 1991). Numerous data obtained for different input powers, temperatures and test time durations indicate the contribution of the present research study compared with previous reported works; by which the same results are reported with higher input power $(300 \mathrm{~W})$ and during longer time of 20 minutes. Besides the reason for avoiding the complexity of doing large number of tests on various types of nuts, this research was only conducted on pistachio nuts, 
because, pistachios are the most suitable substrate being contaminated by the fungus and as mentioned above, pistachio is economically of utmost importance for countries where it is cultivated such as authors' native place.

The sample pistachios used in this research were from Akbari brand which is among the best quality and the most expensive one in Iran. The outer shell of the fruit is green and it turns to red while rippening. The weight of fresh fruit with green shell is 48.41 grams. The wet green and bony shells are weighting 19.3 and 29.8 grams, respectively. The dried fruit along with bony shell is 1.45 grams out of which only 0.75 grams is pistachio brain.

\section{MATERIALS AND METHODS}

The main materials and instruments used in this research study are as followings:

- $\quad$ Pistachio samples; Ten unified pistachios for each test were used Pistachios for each test were surface disinfested to eliminate possible growth of other microorganisms by rinsing them with alcohol.

- $\quad$ Seven spores of Aspergillus flavus having $10^{6}$ Colony Forming Unit per grams (6 logs CFU/gr.) provided by animal husbandry department of Tehran University.

- Yeast Extract Glucose Chloramphenicol (YEGC) Agar product of Merck KGaA, Germany, Code No. 1160000500.

Autoclave 10 litters capacity.

- Laboratory class N.

- $\quad$ Setting up sterilization till $134 \mathrm{C}^{0}$ and setup time is 1 to 59 minutes.

- Incubator with capacity of 55 litters with temperature ranging of -10 to $+45 \mathrm{C}^{0}$ trade mark PECO.

- Plates, plastic/glass containers for placing samples.

- $\quad$ AP-CCP generating device, which was made by authors at concerned laboratory in Plasma and Nuclear Fusion Department, Nuclear Science and Technology Research Institute (NSTRI), Tehran, Iran.

- $\quad$ Peptone water of $0.1 \%$, product of Merck KGaA, Germany, Code No. 1072280500

- Refrigerator, sampler calibrator, metallic sterilized clamps, balance, vibrator, distilled water, alcohol and basher.

\section{Microbial Cultures}

Pure isolate of Aspergillus flavus provided by animal husbandry department of Tehran University, was grass cultured on YEGC Agar plate at Pastor specialized laboratory, Tehran. The plate were kept in an incubator at $37 \mathrm{C}^{0}$ for 24 hours and then at $25 \mathrm{C}^{0}$ for the next 48 hours. A total of 10 nuts were placed on 3 day-old plate and gently rolled around to distribute spores uniformly. In order to increase the fungal contamination level, the sample nuts were incubated on 3 day-old YEGC plates for further 13 hours at $20 \mathrm{C}^{0}$. Next, to eliminate excess moisture the samples were maintained under a laminar flow hood for 3 hours.

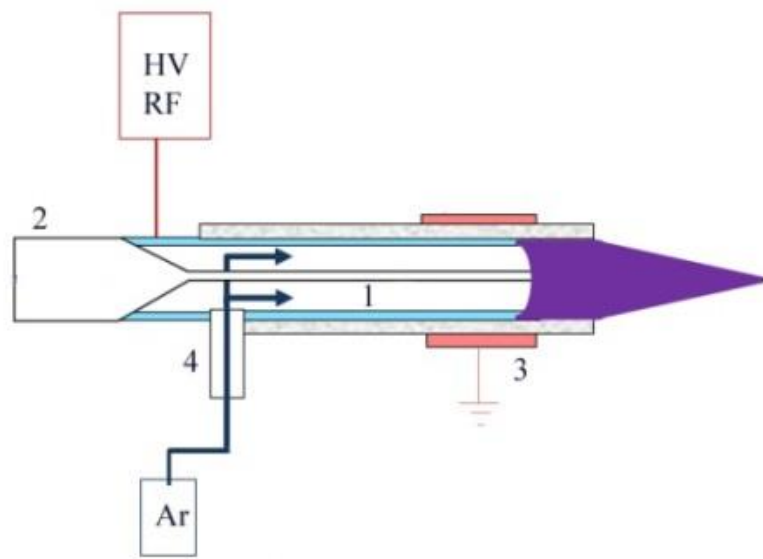

Figure 1 Schematic setup of AP-CCP device: 1- Anode, 2- Body, 3- Cathode, 4Gas entrance

\section{Experimental Device and Process}

The schematic of AP-CCP generating device used in this study is shown in Figure 1 (It consists of a Radio Frequency (RF) power supply machine, Argon gas cylinder, a glass made cylindrical movable container and the AP-CCP generating apparatus which works like a plasma torch (jet)). The AP-CCP apparatus consists of two concentric electrodes through which the working gas flows: one was RF powered and the other was grounded. In order to prevent arcing, a quartz tube was placed between the electrodes. By applying RF power to the inner electrode, the gas discharge is ignited. The ionized gas exits through a nozzle by the flow rate of $50 \mathrm{sccm}$.

The plasma jet was manually controlled to ensure irradiation of plasma all over the object (pistachios), placed in the container. The cable of RF power supply and Argon gas entered from the bottom end of the device. Once gas passing between the dielectrics inside the device, the ionization took place and the plasma was ejected from front end of the device. The experimental device is shown in Figure 2. Frequency of RF machine was $13.65 \mathrm{MHz}$ and it could be set up at $50 \mathrm{ohm}$ on standby mode by using a matching network. The digital indicator showed the variation in power supply. The pistachio irradiation was done very easily by direct exposure of plasma on the surface of samples in atmospheric pressure. The various tests were performed with different input powers of 50, 75, 100 and 150 watts, each one at time durations of 2, 6 and 10 minutes. However, the most favorable results, discussed in this paper, were obtained at 100 watts. To prepare and provide the samples for fungi counting and the final results of each test, the following steps were carefully taken:

In order to obtain a more favorable result, the mixed microbial culture method was used. The colon growth of fungi was observed after the separation process. The colony growth, irradiation and counting process were repeated for each test

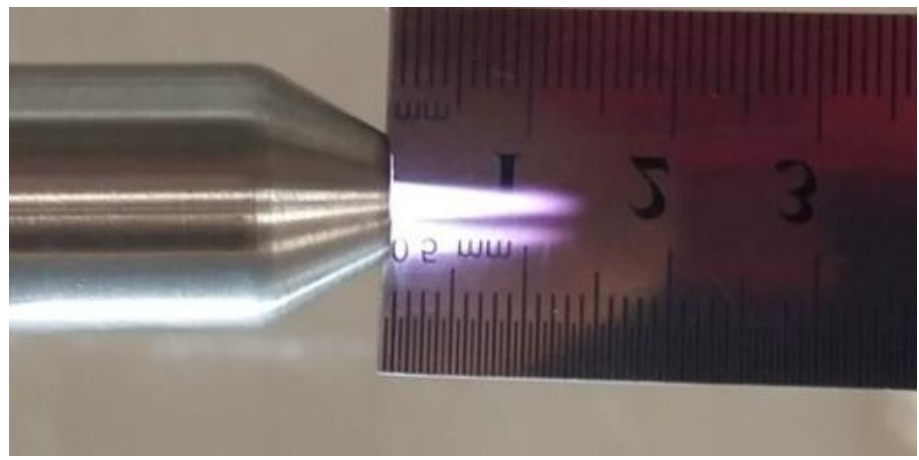

Figure 2 AP-CCP generating device (experimental tool)

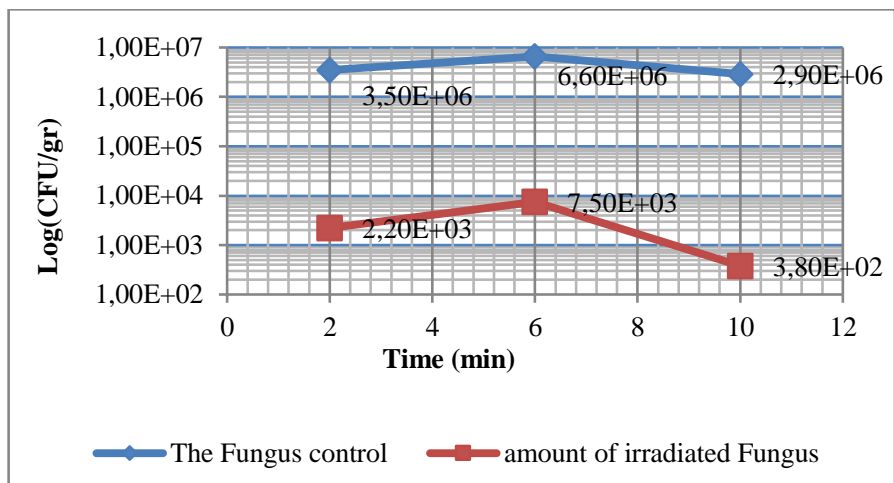

Figure 3 The rate of Fungi reduction by AP-CCP generating device at 100 watts

\section{RESULTS AND DISCUSSION}

After irradiation process with AP-CCP generating device, counting the irradiated sample fungus and also the control fungi count, the results as shown in Table 1 were obtained.

Table 1 shows that pistachios have been affected by plasma, leading to a $3 \log$ fungus reduction, while at 10 minutes irradiation time 4 logs of total fungus (6 $\operatorname{logs}$ ) were reduced. The average of every three tests at three different times of 2, 6 and 10 minutes is, respectively, depicted in Figure 3. It is to be noted that within the laboratory set-ups available for this research, a number of tests were conducted with different input powers of 50, 75, 100, 125 and 150 Watts. At power inputs less than 100 Watts, no fungus reduction was observed even at longer irradiation time and hence those results are not reported in this article. But at 150 Watts, the obtained results at lower time duration were not observably altered, while at 10 minutes irradiation time the fungus was totally reduced (6 logs reduction), but some burnings were observed on the sample shells and the handmade AP-CCP 
device was also heated up. Therefore, due to such uncertainties, the results for power input of 150 Watts are not included in this research report, too. However, it is expected that at large scale industrial application, where the systems can work automatically and probable human inaccuracies are removed, the observed damage on the nut shells may not occur.

\section{Analysis Of Variance (ANOVA) test}

In order to perform the organoleptic analysis of plasma treated samples, a taste panel was organized to compare the sensory quality of pistachio samples before and after 10 minutes irradiation and for both power inputs of 100 and 150 watts, separately. At each stage, a group of 10 experts having no allergic to pistachios were invited and samples including five pistachios of plasma treated were placed in dishes coded with random three digit numbers, and participants were asked to evaluate samples for color, odor, texture, overall appearance and acceptability, using a 1-5 scale. Evaluations were made for each sample with respect to overall acceptance and appearance. The sensory results were evaluated by two factors (panelist and product) ANOVA statistical method. At stage with 100 watts power input, the sensory panelists observed no difference in overall appearance, color, odor and texture but at stage with 150 watts the panelists observed slight alteration only in texture of treated pistachios.

Table 1 Fungi reduction by AP-CCP generating device at 100 watts

\begin{tabular}{ccc}
\hline $\begin{array}{c}\text { Time } \\
(\text { min })\end{array}$ & Fngus control & Irradiated sample \\
\hline 2 & $3.3 \times 10^{6}$ & $1.9 \times 10^{3}$ \\
\hline 2 & $3.9 \times 10^{6}$ & $2.2 \times 10^{3}$ \\
\hline 2 & $3.4 \times 10^{6}$ & $2.5 \times 10^{3}$ \\
\hline 6 & $6.4 \times 10^{6}$ & $6.7 \times 10^{3}$ \\
\hline 6 & $6.7 \times 10^{6}$ & $8.2 \times 10^{3}$ \\
\hline 6 & $4.8 \times 10^{6}$ & $7.6 \times 10^{3}$ \\
\hline 10 & $3.1 \times 10^{6}$ & $2.9 \times 10^{2}$ \\
\hline 10 & $3.3 \times 10^{6}$ & $4.4 \times 10^{2}$ \\
\hline 10 & $2.5 \times 10^{6}$ & $4.1 \times 10^{2}$ \\
\hline
\end{tabular}

\section{CONCLUSION}

In comparison with other conventional methods such as: chemicals, physical and biological farming techniques (; Takahashi et al., 2002; Goryacheva et al., 2007; Rashid et al., 2008; Gupta et al., 2012; Hell et al., 2010; Tsitsigiannis et al. 2012), and supporting the earlier research reports, the present research work proved that using cold plasma is one of the best options for treatment, inactivation and/or acceptable reduction of fungus Aspergillus flavus amount from nut shells, preventing further toxin production in nuts; in general and particularly in pistachios.

In order to reduce the fungus Aspergillus flavus from pistachios, and for the first time an AP-CCP generating device was used in this research. Various tests were carried out, using Argon gas as plasma forming gas in atmospheric pressure with different input power and irradiation times of 2,6 and 10 minutes respectively. The acceptable amount of $4 \log$ fungus reduction (about 67\%) was obtained at power input of $100 \mathrm{~W}$ for 10 minutes irradiation time with no alteration on the nut texture Therefore, this amount of fungus reduction is considered as final and the confirmed result of this research report. It is to be mentioned that the same test with $150 \mathrm{~W}$ in 10 minutes was also carried out and the fungus was totally $(100 \%)$ removed. But since the surface of pistachios, treated by plasma generated by power inputs above 100 watts, were slightly altered in texture only and due to device heating; such results are not declared as confirmed achievements of this research work. However, the authors believe that at big scale industrial application, where the devices would easily bear higher input power, the present deficiency may not occur.

Nuts in general and particularly pistachios are of utmost economic importance in view of earning foreign currencies from import/export of these energetic agricultural products. The prevailing fungus Aspergillus flavus is one of the most acute and the biggest threat to nuts health. The worldwide stringent imposed regulations in this regard are some of unavoidable hazards in the exports/imports of pistachios. To date, no remedies which could be applied in big industrial scale has not been reported. Hence, it is hoped that the results of this research could encourage the related industries to use the developed method in order to solve this global problem and prevent the related annual economic losses.

\section{REFERENCES}

Arrus, K., Blank, G., Abramson, D., Clear, R., \& Holley, R. A. (2005). Aflatoxin production by Aspergillus flavus in Brazil nuts. Journal of Stored Products Research, 41(5), 513-527.http://dx.doi.org/10.1016/j.jspr.2004.07.005

Martins, H. M., Almeida, I., Marques, M., \& Bernardo, F. (2008). Interaction of wild strains of Aspergilla with Aspergillus parasiticus ATCC15517 on aflatoxins production. International journal of molecular sciences, 9(3), 394-400. http://dx.doi.org/10.3390/ijms9030394

Gourama, H., \& Bullerman, L. B. (1995). Aspergillus flavus and Aspergillus parasiticus: aflatoxigenic fungi of concern in foods and feeds: a review. Journal of Food protection, 58(12), 1395-1404. http://dx.doi.org/ 10.4315/0362-028X58.12.1395

Petitpas, G., Rollier, J. D., Darmon, A., Gonzalez-Aguilar, J., Metkemeijer, R., \& Fulcheri, L. (2007). A comparative study of non-thermal plasma assisted reforming technologies. International Journal of Hydrogen Energy, 32(14), 2848-2867. http://dx.doi.org/ 10.1016/j.ijhydene.2007.03.026

Lieberman, M. A., \& Lichtenberg, A. J. (2005). Principles of plasma discharges and materials processing. John Wiley \& Sons. http://dx.doi.org/ $10.1002 / 0471724254$

Roth, J. R., Sherman, D. M., Gadri, R. B., Karakaya, F., Chen, Z., Montie, T. C., \& Tsai, P. Y. (2000). A remote exposure reactor (RER) for plasma processing and sterilization by plasma active species at one atmosphere. IEEE Transactions on Plasma Science, 28(1), 56-63. http://dx.doi.org/ 10.1109/27.842864

Gadri, R. B., Roth, J. R., Montie, T. C., Kelly-Wintenberg, K., Tsai, P. P. Y., Helfritch, D. J., ... \& Team, U. P. S. (2000). Sterilization and plasma processing of room temperature surfaces with a one atmosphere uniform glow discharge plasma (OAUGDP). Surface and Coatings Technology, 131(1), 528-541. https://doi.org/10.1016/S0257-8972(00)00803-3

Ohkawa, H., Akitsu, T., Tsuji, M., Kimura, H., Kogoma, M., \& Fukushima, K. (2006). Pulse-modulated, high-frequency plasma sterilization at atmosphericpressure. Surface and coatings technology, 200(20), 5829-5835 https://doi.org/10.1016/j.surfcoat.2005.08.124

Lee, K., Paek, K. H., Ju, W. T., \& Lee, Y. (2006). Sterilization of bacteria, yeast, and bacterial endospores by atmospheric-pressure cold plasma using helium and oxygen. The Journal of Microbiology, 44(3), 269-275.

Park, B. J., Takatori, K., Sugita-Konishi, Y., Kim, I. H., Lee, M. H., Han, D. W. .. \& Park, J. C. (2007). Degradation of mycotoxins using microwave-induced argon plasma at atmospheric pressure. Surface and Coatings Technology, 201(9), 5733-5737. https://doi.org/10.1016/j.surfcoat.2006.07.092

Samarajeewa, U. (1991). In situ degradation of mycotoxins by physical methods. Mycotoxins and Animal Foods, 785-796.

Schneider, J., Baumgärtner, K. M., Feichtinger, J., Krüger, J., Muranyi, P., Schulz, A., ... \& Schumacher, U. (2005). Investigation of the practicability of low-pressure microwave plasmas in the sterilisation of food packaging materials at industria level. Surface and coatings technology, 200(1), 962-966 https://doi.org/10.1016/j.surfcoat.2005.01.114

Moisan, M., Barbeau, J., Crevier, M. C., Pelletier, J., Philip, N., \& Saoudi, B. (2002). Plasma sterilization. Methods and mechanisms. Pure and applied chemistry, 74(3), 349-358. http://dx.doi.org/10.1351/pac200274030349

Basaran, P., Basaran-Akgul, N., \& Oksuz, L. (2008). Elimination of aspergillus parasiticus from nut surface with low pressure cold plasma (LPCP) treatment. Food Microbiology, 25(4), 626-632. http://dx.doi.org/10.1016/j.fm.2007.12.005

Uhm, H. S., Lim, J. P., \& Li, S. Z. (2007). Sterilization of bacterial endospores by an atmospheric-pressure argon plasma jet. Applied physics letters, 90(26), 261501 http://dx.doi.org/10.1063/1.2747177

Gupta, M., Manisha, K., \& Grover, R. (2012). Effect of various media types on the rate of growth of Aspergillus niger. Indian Journal of Fundamental and Applied Life Sciences, 2(2), 141-144. http://www.cibtech.org/jls.htm

Takahashi, T., Chang, P. K., Matsushima, K., Yu, J., Abe, K., Bhatnagar, D., ... \& Koyama, Y. (2002). Nonfunctionality of Aspergillus sojae aflR in a strain of Aspergillus parasiticus with a disrupted aflR gene. Applied and environmenta microbiology, 68(8), 3737-3743. http://dx.doi.org/10.1128/AEM.68.8.37373743.2002

Rashid, M. A. L. I. H. A., Khalil, S. A. M. I. N. A., Ayub, N., Ahmed, W. A. S. E. E. M., \& Khan, A. G. (2008). Categorization of Aspergillus flavus and Aspergillus parasiticus isolates of stored wheat grains in to aflatoxinogenics and nonaflatoxinogenics. Pakistan Journal of Botany, 40(5), 2177-2192.

Gqaleni, N., Smith, J. E., Lacey, J., \& Gettinby, G. (1997). Effects of temperature, water activity, and incubation time on production of aflatoxins and cyclopiazonic 
acid by an isolate of Aspergillus flavus in surface agar culture. Applied and environmental microbiology, 63(3), 1048-1053.

Goryacheva, I. Y., Saeger, S. D., Eremin, S. A., \& Peteghem, C. V. (2007) Immunochemical methods for rapid mycotoxin detection: Evolution from single to multiple analyte screening: A review. Food additives and contaminants, 24(10), 1169-1183. http://dx.doi.org/10.1080/02652030701557179

Tsitsigiannis, D. I., Dimakopoulou, M., Antoniou, P. P., \& Tjamos, E. C. (2012). Biological control strategies of mycotoxigenic fungi and associated mycotoxins in Mediterranean basin crops. Phytopathologia Mediterranea, 158-174. http://dx.doi.org/10.14601/Phytopathol_Mediterr-9497

Hell, K., Mutegi, C., \& Fandohan, P. (2010). Aflatoxin control and prevention strategies in maize for Sub-Saharan Africa. Julius-Kühn-Archiv, (425), 534. http://dx.doi.org/10.5073/jka.20100425.388 